\title{
High-birefringence direct-UV-written silica waveguides for heralded single-photon sources at telecom wavelengths
}

\author{
M. T. Posner, R. H. S. Bannerman, D. H. Smith, P. L. Mennea, J. C. Gates, and P. G. R. Smith \\ Optoelectronics Research Centre, University of Southampton, Southampton, Hampshire, SO17 1BJ, U.K.
}

The reliable generation of high-quality single photons has long been a goal of research in photonic quantum information. Direct-UV-written (DUW) silica-on-silicon waveguides provide a reproducible, uniform, low-loss, and fiber-compatible platform for the integration of spontaneous four-wave mixing-based heralded single-photon sources [1]. To overcome limitations caused by Rayleigh scattering, which induces loss, and for compatibility with telecommunications equipment, it is desirable to move to the C-band. Waveguides with birefringence greater than $4.5 \times 10^{-4}$ are required in this wavelength of operation [2]. We report the development of a highbirefringence silica-on-silicon integrated platform for heralded single-photon sources at telecom wavelengths.

Silica-on-silicon wafers for DUW waveguides are usually fabricated by flame hydrolysis deposition (FHD) by the consecutive deposition and consolidation of silicate soot layers - Fig. 1(a). These ternary silicate systems have received much attention from a production perspective, but there is little literature on methods to increase the birefringence in waveguides. It is well understood that a reduction in the concentration of germanium and boron dopants in silica layers leads to an increase in the glass melting point. This change results in an increase of the expansion coefficient mismatch between the layers causing additional stress and increased birefringence. A characteristic of DUW waveguides is the conservation of the birefringence of the layer compared to their etched counterparts; previous waveguide devices fabricated by flame hydrolysis deposition (FHD) and DUW show birefringence on the order $2-3 \times 10^{-4}$. The reduction in boron and germanium dopants also reduces the glass photosensitivity in the UV and modifies the refractive index. We have fabricated an initial set of devices to study the compatibility of this material platform with DUW and assess its suitability for applications in quantum optics.

Our devices are fabricated on substrates comprised of a $16 \mu \mathrm{m}$ thermal oxide grown onto a silicon wafer. A planar core is deposited by FHD with concentrations of germanium and boron dopants lower than previously reported. A subsequent FHD step is performed to deposit a cladding. We have fabricated silica waveguides and gratings simultaneously by DUW [3] at a range of fluence from 12-24 kJ/cm² with Bragg gratings at resonant wavelengths from $1545-1570 \mathrm{~nm}$.

(a)

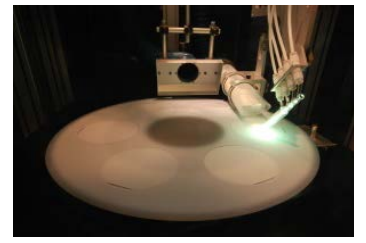

(b)

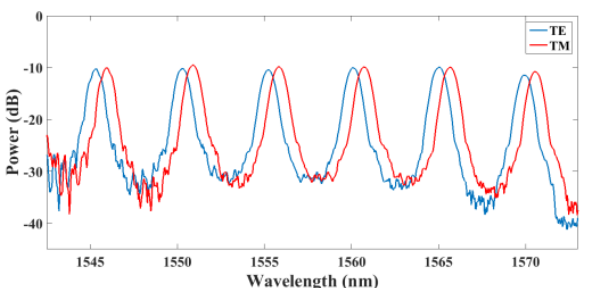

(c)

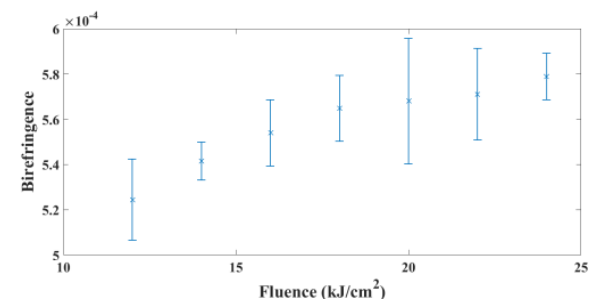

Fig. 1 (a) Photo of the FHD system used to fabricate high-birefringence silicate DUW waveguides. (b) Representative Bragg grating reflection spectrum for TE and TM mode of fabricated devices. (c) Average waveguide birefringence variation with respect to fluence obtained through grating reflection spectrum analysis; error bars show standard deviation of the birefringence for each set of Bragg gratings in the corresponding waveguide.

The waveguides are characterized following an in-situ grating-based measurement technique [4]. Typical grating reflection spectra for orthogonal TE and TM polarizations are shown in Fig. 1(b). The waveguide birefringence is calculated from the wavelength shift in the Bragg reflections for orthogonal polarization states for each grating. Waveguide birefringence from $5.24 \pm 0.18 \times 10^{-4}$ to $5.79 \pm 0.10 \times 10^{-4}$ have been measured across a range of fluence as shown in Fig. 1(c). The data shows that the birefringence of the waveguides can be finetuned by controlling the fluence. These preliminary results show that the necessary birefringence values required for heralded single-photon sources at telecom wavelengths are achievable on our silica-on-silicon platform. We will report on the fabrication process with emphasis on the requirements needed for integration of on-chip single-photon sources. We will provide detail on the experimental methods for the characterization of these devices in terms of birefringence, uniformity and loss.

\section{References}

[1] J. B. Spring, et. al., “Chip-based array of near-identical, pure, heralded single-photon sources,” Optica, vol. 4, no. 1, pp. 90-96, 2017.

[2] B. J. Smith, et. al., "Photon pair generation in birefringent optical fibers," Opt. Express, vol. 17, no. 26, pp. 23589-23602, 2009.

[3] C. Sima, et. al., "Ultra-wide detuning planar Bragg grating fabrication technique based on direct UV grating writing with electro-optic phase modulation,” Opt. Express, vol. 21, no. 13, pp. 15747-15754, 2013.

[4] H. L. Rogers, et. al., "In situ loss measurement of direct UV-written waveguides using integrated Bragg gratings,” Opt. Lett., vol. 35, no. 17 , pp. 2849-2851, 2010. 